\title{
A PEST sequence in ABCA1 regulates degradation by calpain protease and stabilization of ABCA1 by apoA-I
}

\author{
Nan Wang, Wengen Chen, Patrick Linsel-Nitschke, Laurent O. Martinez, \\ Birgit Agerholm-Larsen, David L. Silver, and Alan R. Tall \\ Division of Molecular Medicine, Department of Medicine, Columbia University, New York, New York, USA
}

\begin{abstract}
Cholesterol-loaded macrophage foam cells are a central component of atherosclerotic lesions. ABCA1, the defective molecule in Tangier disease, mediates the efflux of phospholipids and cholesterol from cells to apoA-I, reversing foam cell formation. In ABCA1, we identified a sequence rich in proline, glutamic acid, serine, and threonine (PEST sequence) that enhances the degradation of ABCA 1 by calpain protease and thereby controls the cell surface concentration and cholesterol efflux activity of ABCA1. In an apparent positive feedback loop, apoA-I binds ABCA1, promotes lipid efflux, inhibits calpain degradation, and leads to increased levels of ABCA1. ApoA-I infusion also increases ABCA1 in vivo. These studies reveal a novel mode of regulation of ABCA1 by PEST sequence-mediated calpain proteolysis that appears to be reversed by apolipoprotein-mediated phospholipid efflux. Inhibition of ABCA1 degradation by calpain could represent a novel therapeutic approach to increasing macrophage cholesterol efflux and decreasing atherosclerosis.
\end{abstract}

J. Clin. Invest. 111:99-107 (2003). doi:10.1172/JCI200316808.

\section{Introduction}

Atherosclerosis, the leading cause of morbidity and death in industrialized societies, is initiated by the deposition of lipoprotein cholesterol in the artery wall. After retention and modification in arteries, atherogenic lipoproteins are taken up by macrophages, giving rise to cholesterol-engorged foam cells (1). Elevated levels of HDL are associated with a decrease in atherosclerosis that is believed to be due in part to the ability of HDL and its apolipoproteins to remove cholesterol from foam cells $(2,3)$. The infusion or transgenic overexpression of the HDL-associated apolipoproteins apoA-I or apoE decreases atherosclerosis in animals (4-6). This has led to human clinical trials in which apoA-I or variants, such as apoA-I $\mathrm{I}_{\text {Milano, }}$, are being infused intravenously as a treatment for atherosclerosis $(7,8)$. However, there is uncertainty as to the underlying mechanisms of protection, and it is debated whether HDL is acting to promote reverse cholesterol transport, by anti-inflammatory or antithrombotic effects, and even whether it is merely a metabolic marker for a tendency to accumulate atherogenic remnants of triglyceride-rich lipoproteins $(9,10)$. In Tangier disease, very low plasma HDL levels are associated with macrophage foam cell accumulation in

Received for publication September 3, 2002, and accepted in revised form November 11, 2002.

Address correspondence to: Nan Wang, Division of Molecular Medicine, Department of Medicine, Columbia University, 630 West 168th Street, New York, New York 10032, USA. Phone: (212) 305-5789; Fax: (212) 305-5052;

E-mail: nw30@columbia.edu.

Conflict of interest: The authors have declared that no conflict of interest exists.

Nonstandard abbreviations used: ATP binding cassette transporter A1 (ABCA1); liver X receptor (LXR); retinoid X receptor (RXR). various organs such as the spleen or tonsils and with an excess of atherosclerosis (11). Tangier disease is caused by mutations in ATP-binding cassette transporter A1 (ABCA1). ABCA1 mutations also are a cause of familial isolated low HDL and are associated with premature atherosclerosis $(12,13)$. ABCA1 in macrophages, hepatocytes, and other cells mediates phospholipid and cholesterol efflux to extracellular apoA-I and forms nascent HDL (14), which absorbs further lipids as it matures in the bloodstream and eventually returns its cholesterol to the liver. Thus, the upregulation of ABCA1 expression may provide a key to HDL formation and promotion of foam cell cholesterol efflux (15).

The cellular expression of ABCA1 is highly regulated. In macrophages $A B C A 1$ protein is undetectable in the basal state, but expression is markedly increased with cholesterol loading, as a result of transcription stimulated by liver $X$ receptor (LXR) and retinoid $X$ receptor (RXR) $(15,16)$. In human monocyte-derived macrophages loaded with acetyl-LDL cholesterol esters, the increase of ABCA1 protein appears disproportionate to the induction of ABCA1 mRNA (17), and the turnover of $A B C A 1$ protein in macrophages is rapid $(18,19)$. These observations suggest that $A B C A 1$ protein turnover could play a role in regulation of ABCA1 function. ABCA1 binds and cross-links apoA-I $(14,18)$, raising the possibility that apoA-I modulates the turnover of ABCA1. Indeed, a recent study has revealed that binding of apoA-I and apoA-II to ABCA1 in human THP1 macrophages increases levels of ABCA1 by protecting it from degradation by an unknown thiol protease (19). In this study we show that $A B C A 1$ protein degradation is regulated by a PEST sequence in ABCA1 and is mediated by calpain protease. In a novel form of positive feedback control, the interaction of ABCA1 with apoA-I leads to inhibi- 
tion of calpain protease degradation and an increase in $A B C A 1$ protein at the cell surface.

\section{Methods}

Chemicals and reagents. Human apoA-I (BIODESIGN International, Saco, Maine, USA) was dialyzed against PBS. M2 anti-FLAG antibody was from (St. Louis, Missouri, USA); PD8407 anti-ubiquitin antibody was from Santa Cruz Biotechnology Inc. (Santa Cruz, California, USA); rabbit polyclonal anti-ABCA1 antibody was from Novus Biologicals Inc. (Littleton, Colorado, USA). Purified $\mu$-calpain, calpeptin, lactacystin, and N-Ac-LeuLeu-norleucinal (ALLN) were from Calbiochem-Novabiochem Corp. (San Diego, California, USA).

Plasmid constructs and cell transfection. ABCA1-FLAG was constructed as described (14). ABCA1delPEST was based on ABCA1-FLAG. Using PCR, we constructed ABCA1delPEST by deleting a nucleotide sequence that encodes mouse ABCA1 amino acids 1283-1306 and confirmed it by sequencing. HEK293 cells, in 12- or 24well collagen-coated plates, were transiently transfected with various plasmid constructs at indicated DNA concentrations with Lipofectamine 2000 (Invitrogen Corp., San Diego, California, USA) at $37^{\circ} \mathrm{C}$ overnight ( 20 hours). We routinely used a construct expressing green fluorescence protein (GFP) to visually monitor transfection efficiency (i.e., the percentage of cells expressing GFP). The transfection efficiency of 293 cells was in the range of $50-80 \%$ of cells. Although transfection efficiency did vary from experiment to experiment, we found that the variation within the same experiment was small (generally less than $10 \%$ ). In addition to performing multiple replicates within each experiment, we repeated all experiments on multiple separate occasions to confirm reproducibility of results.

Cellular lipid efflux assays, apoA-I cell association, and chemical cross-linking. The assays were carried out as in ref. 14. Generally, 293 cells were labeled by culturing overnight in media containing either $\left[{ }^{3} \mathrm{H}\right]$ cholesterol, for cholesterol efflux, or $\left[{ }^{3} \mathrm{H}\right]$ choline, for phospholipid efflux. The next day, cells were washed with fresh media before or after treatment as indicated, and then apoA-I was added as acceptor and incubated for the indicated period before the media and cells were collected for analysis. Mouse peritoneal macrophage cells were isolated from male mice by peritoneal lavage with PBS 3 days after intraperitoneal injection with $1 \mathrm{ml}$ of $3.85 \%$ thioglycollate. The cells were labeled with $1 \mathrm{mCi} / \mathrm{ml}$ $\left[{ }^{3} \mathrm{H}\right]$ cholesterol overnight in DMEM and $0.2 \%$ BSA supplemented with $50 \mu \mathrm{g} / \mathrm{ml}$ acetylated LDL plus LXR/ RXR ligands 22(R)-hydroxycholesterol and 9-cis retinoic acid (both $10 \mu \mathrm{M}$ ). After labeling, cells were washed and efflux was carried out with $10 \mu \mathrm{g} / \mathrm{ml}$ apoA-I for 3 hours. Then, cells and medium were collected for analysis. Cholesterol efflux was expressed as the percentage of the radioactivity released from the cells into the medium, relative to the total radioactivity in cells plus medium. For apoA-I cell association, cells were incubated with $0.2 \mu \mathrm{g} / \mathrm{ml}\left[{ }^{125} \mathrm{I}\right] \mathrm{apoA}-\mathrm{I}$ in $0.2 \% \mathrm{BSA}$ and
DMEM for 1 hour at $37^{\circ} \mathrm{C}$. After being washed three times with fresh media, cells were lysed with $0.1 \%$ SDS and $0.1 \mathrm{~N} \mathrm{NaOH}$ lysis buffer, and radioactivity was determined by a gamma counter.

Immunoprecipitation and immunoblot analysis of $A B C A 1$. For immunoblot analysis of ABCA1, ABCA1-FLAG, and ABCA1delPEST-FLAG, transfected HEK293 cells, peritoneal macrophages, or primary hepatocytes were washed and scraped in PBS and lysed in RIPA buffer (10 $\mathrm{mM}$ Tris- $\mathrm{HCl}\left[\mathrm{pH}\right.$ 7.3], $1 \mathrm{mM} \mathrm{MgCl}_{2}, 1.0 \%$ Nonidet P-40, $0.5 \%$ sodium deoxycholate, and $5 \mathrm{mM}$ EDTA in the presence of protease inhibitors as follows: $0.5 \mu \mathrm{g} / \mathrm{ml}$ leupeptin, $1 \mu \mathrm{g} / \mathrm{ml}$ aprotinin, and $1 \mu \mathrm{g} / \mathrm{ml}$ pepstatin A) (Roche Molecular Biochemicals, Indianapolis, Indiana, USA). Postnuclear supernatants containing the indicated amounts of protein were subjected to Western analysis using an anti-ABCA1 antiserum or anti-FLAG M2 antibody and chemiluminescence detection. The relative intensities of the bands were determined by densitometry. For cell surface ABCA1 analysis, cells were first biotinylated with $0.5 \mathrm{mg} / \mathrm{ml}$ EZ-Link Sulfo-NHSSS-Biotin (Pierce Chemical Co., Rockford, Illinois, USA) at $4^{\circ} \mathrm{C}$ for 30 minutes. Then cells were lysed with RIPA buffer at $4^{\circ} \mathrm{C}$. After centrifugation, the supernatant of cell lysates was incubated with anti-FLAG agarose beads overnight at $4{ }^{\circ} \mathrm{C}$. Following centrifugation and washing, the collected agarose beads were subjected to SDSPAGE sample buffer with $100 \mathrm{mM}$ 2-mercaptoethanol. The total or ubiquitinated ABCA1 was detected by Western blot using either anti-FLAG antibody or streptavidin-horseradish peroxidase.

Calpain-catalyzed proteolysis of metabolically labeled ABCA1. Transiently transfected 293 cells were pulselabeled by $\left[{ }^{35} \mathrm{~S}\right]$ methionine $(0.5 \mathrm{mCi} / \mathrm{ml})$ in $0.2 \% \mathrm{BSA}$ and DMEM for 2 hours. Cells were washed three times with fresh media and placed on ice for 10 minutes. Then cells were permeabilized by addition of $80 \mu \mathrm{g} / \mathrm{ml}$ digitonin in DMEM and incubated on ice for 15 minutes. Next, the cells were washed twice with fresh DMEM, and then purified $\mu$-calpain in DMEM plus 2 $\mathrm{mM} \mathrm{CaCl}_{2}$ was added at the indicated concentration and incubated for 20 minutes at room temperature. Then cells were lysed by addition of $1 \mathrm{ml}$ RIPA buffer with $40 \mu \mathrm{g} / \mathrm{ml}$ calpeptin. FLAG-tagged ABCA1 was immunoprecipitated by anti-FLAG agarose beads and subjected to quantitative analysis by PhosphorImager.

Primary hepatocytes. Hepatocytes were isolated according to Honkakoski and Negishi (20), except that complete protease inhibitor was added to digestion buffer according to the manufacturer's instructions (Roche Molecular Biochemicals).

Apolipoprotein A-I infusion in mice. All mice used in these studies were 8-week-old female wild-type mice of the inbred strain C57BL/6J and were fed a chow diet. Mice were anesthetized intraperitoneally with 0.1 $\mathrm{ml} / 30 \mathrm{~g}$ body weight of a solution containing 100 $\mathrm{mg} / \mathrm{ml}$ ketamine and $30 \mathrm{mg} / \mathrm{ml}$ xylazine. ApoA-I (20 $\mathrm{mg} / \mathrm{kg}$ body weight) or BSA as control was administered via femoral vein as a bolus injection. Four hours 
after injection, animals were euthanized and the liver was dissected out for analysis.

\section{Results}

A PEST sequence in ABCA1 modulates ABCA1 degradation. Using metabolic labeling in transfected 293 cells, we showed that ABCA1 protein turns over rapidly, with a half-life of approximately 1 hour (not shown). Many proteins that undergo rapid turnover contain sequences enriched in proline, glutamic acid, serine, and threonine, called PEST sequences (21). Using the program PESTfind (22), we identified a conserved potential PEST sequence in ABCA1 (Figure 1a; PEST score, +16.42 ; PEST scores greater than +5 are considered significant). In order to evaluate the role of the PEST sequence, we expressed a FLAG-tagged PEST deletion mutant (ABCA1delPEST) in 293 cells. As shown by biotinylation and immunoprecipitation, cell surface levels of ABCA1delPEST were markedly increased compared with wild-type ABCA1 (Figure 1b, top; mean fold increase $3.9 \pm 0.4, n=4, P<0.001)$. In contrast, overall expression levels of ABCA1delPEST in cell lysates were slightly lower than those of wild-type ABCA1 (Figure $1 \mathrm{~b}$, bottom), reflecting a decreased rate of synthesis of the deletion mutant (70\% of wild-type as determined by metabolic pulse-labeling using $\left[{ }^{35} \mathrm{~S}\right]$ methionine). To assess the function of ABCA1delPEST, we measured cellular binding of apoA-I and phospholipid and cholesterol efflux to apoA-I. All parameters were increased for the ABCA1delPEST mutant compared with wild-type ABCA1 (Figure 1, c, f, and g). When normalized for protein expression levels in cell lysates (Figure 1d), the ABCA1delPEST displayed a two- to fourfold increase in cholesterol efflux (Figure 1e). The effect was larger at lower DNA transfection levels (Figure 1e), suggesting the existence of an unknown rate-limiting factor regulating PEST sequence-mediated ABCA1 turnover. Thus, deletion of the PEST sequence results in increased cell surface concentration of ABCA1, with a proportionate increase in functional activity. These findings indicate that the PEST sequence has a key role in determining the cell surface expression of functional ABCA1 and suggest that the major active form of $A B C A 1$ resides on the cell surface. a

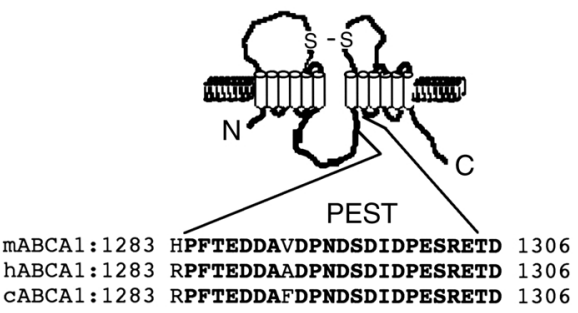

c

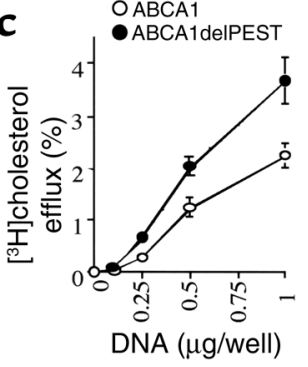

f

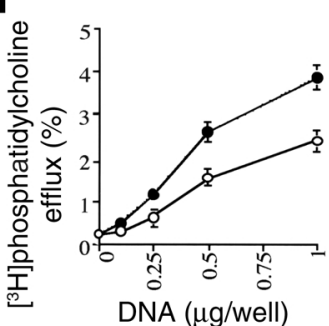

d

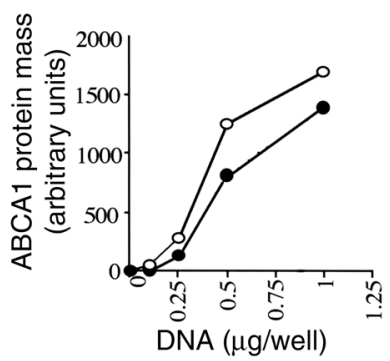

g

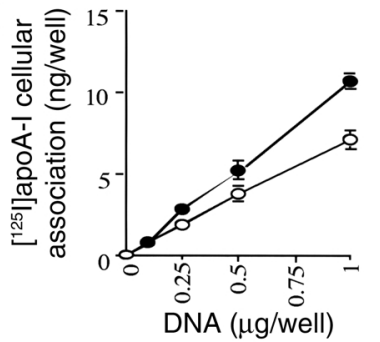

b

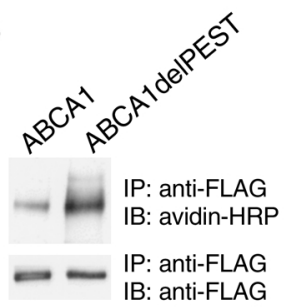

e

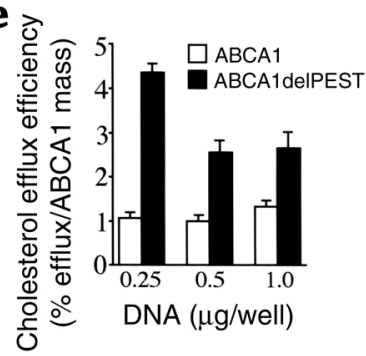

h

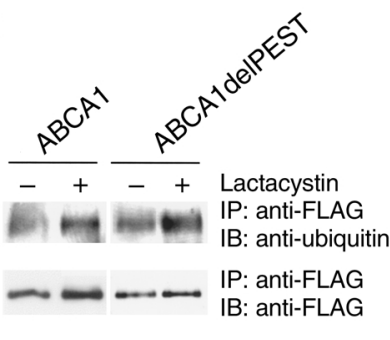

Figure 1

A PEST sequence regulates the cell surface expression and function of ABCA1. (a) Schematic model of ABCA1 showing the location and amino acid sequence of the PEST sequence in mouse $(\mathrm{m})$, human $(\mathrm{h})$, and chicken $(\mathrm{c})$. The PEST score is +16.42 , as determined by PESTfind. (b) Levels of total (bottom) and cell surface (top, biotinylated) ABCA1 and ABCA1delPEST in transiently transfected HEK293 cells. (c, $\mathbf{f}$, and $\mathbf{g}$ ) DNA dose-dependent ABCA1-mediated and ABCA1delPEST-mediated cellular cholesterol efflux (c), phospholipid efflux (f), and apoA-I cell association ( $\mathbf{g}$ ). (d) Total cellular ABCA1 protein mass as determined by quantitative Western blotting in the cholesterol efflux assay. (e) Cholesterol efflux efficiency determined by percentage efflux normalized for total cellular ABCA1 protein mass. (h) Levels of total (bottom) and ubiquitinated (top) ABCA1 and ABCA1delPEST in HEK293 cells incubated with or without lactacystin $(20 \mu \mathrm{M})$ for 3 hours. The data are representative of two experiments ( $\mathbf{c}$ and $\mathbf{e}-\mathbf{h}$, triplicate; $\mathbf{d}$, duplicate) or three experiments (b, duplicate and triplicate) with similar results. 


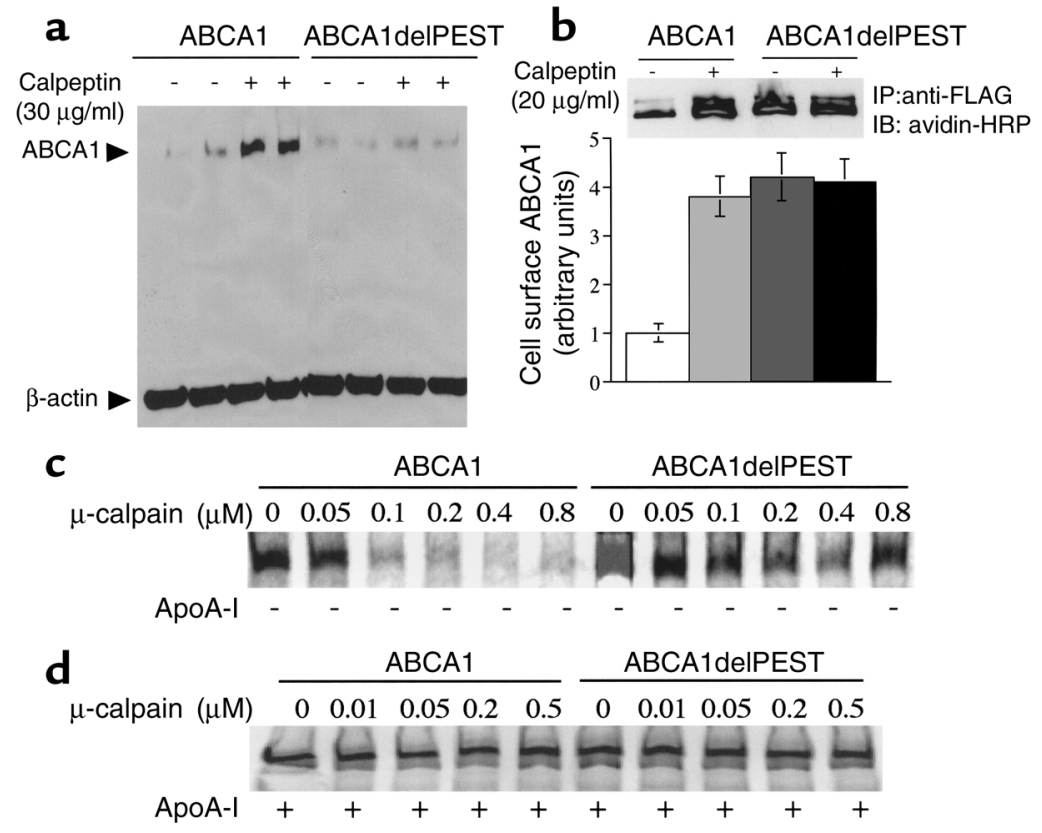

\section{Figure 2}

Calpain protease catalyzes PEST-dependent degradation of ABCA1. (a) Levels of ABCA1 and ABCA1delPEST in 293 cells, incubated with or without calpeptin $(30 \mu \mathrm{g} / \mathrm{ml})$ for 3 hours, as determined by Western blot analysis. (b) Cell surface ABCA 1 or ABCA1delPEST in 293 cells with or without incubation with calpeptin $(20 \mu \mathrm{g} / \mathrm{ml})$ for 3 hours, as determined by biotinylation, immunoprecipitation with anti-FLAG antibody, and Western analysis with avidin-horseradish peroxidase. The data are representative of two experiments, each performed in triplicate, with similar results. (c and d) The susceptibility to exogenously added $\mu$-calpain of ABCA1 or ABCA1 delPEST metabolically labeled by $\left.{ }^{35} \mathrm{~S}\right]$ methionine in digitonin-permeabilized 293 cells with (d) or without (c) pretreatment with apoA-I $(10 \mu \mathrm{g} / \mathrm{ml})$ for 3 hours.
PEST sequences in plasma membrane proteins often increase protein turnover by enhancing the binding of ubiquitin ligase, leading to ubiquitination, endocytosis, and proteasomal degradation of the target molecule (23). We found that ABCA1 was ubiquitinated (Figure 1h) and initially considered a possible role of the PEST sequence in mediating this effect. However, wild-type and PEST deletion ABCA1 were ubiquitinated at similar levels. Lactacystin, a specific inhibitor of proteasomal degradation, caused a similar 1.4- to 1.5fold increase in levels of wild-type and PEST deletion ABCA1 and a 1.8- and a 2.4-fold increase in the corresponding ubiquitinated forms. Similar observations were made in three separate experiments. Although there is ubiquitination and proteasomal degradation of ABCA1, ubiquitination of ABCA1 is not controlled by the PEST sequence (Figure $1 \mathrm{~h}$ ).

PEST-dependent ABCA1 degradation is mediated by calpain protease. In a few instances, PEST sequences have been implicated in proteolysis of membrane proteins by calpains, helping to bind these proteases to their targets $(21,24)$. As in a recent report (19), we found that turnover of ABCA1 protein was decreased by thiol protease inhibitors ALLN, MG132, leupeptin, and E64 (not shown). However, these inhibitors are nonspecific and do not differentiate between different classes of thiol proteases. To see whether ABCA1 might be specifically targeted by calpains, we treated cells with the specific calpain protease inhibitor calpeptin (25). This resulted in a $2.8 \pm 0.3$-fold increase $(n=8 P<0.001)$ in total ABCA1 protein levels (Figure $2 \mathrm{a}$ ), and a $3.7 \pm 0.4$-fold increase $(P=0.005)$ in cell surface ABCA1 (Figure $2 b)$. The effect of calpeptin on ABCA1 in cell lysates was abolished by deletion of the PEST sequence (Figure $2 \mathrm{a}$ ). Moreover, a similar fourfold increase in cell surface ABCA1 was observed with calpeptin treatment or dele- tion of the PEST sequence, and there was no effect of calpeptin on levels of cell surface PEST deletion mutant (Figure 2b). These experiments suggest that ABCA1 is a target of calpain proteolysis, and that the PEST sequence helps to target calpain to cell surface ABCA1.

In order to confirm the role of calpain in degradation of ABCA1 and the involvement of the PEST sequence, cells were permeabilized, washed, and then treated with purified $\mu$-calpain protease, a ubiquitously expressed subtype of calpain proteases. For wild-type ABCA1, this treatment resulted in efficient degradation (Figure 2c). However, there was no appreciable degradation of ABCA1delPEST (Figure 2c), providing direct evidence that PEST-dependent degradation of ABCA1 is mediated by calpain protease.

In order to see whether calpain protease degrades ABCA1 under more physiological conditions as well (i.e., without overexpression of ABCA1 by cell transfection), we treated primary cultures of resident mouse peritoneal macrophages or primary mouse hepatocytes with calpeptin. This also resulted in an increase in ABCA1 protein level (Figure 3, a and b; for macrophages, ABCA1 was increased $3.02 \pm 1.12$-fold, $P<0.05$, at $20 \mu \mathrm{g} / \mathrm{ml}$ calpeptin; for hepatocytes, ABCA1 was increased $2.29 \pm 0.27$-fold, $P<0.05$, at 15 $\mu \mathrm{g} / \mathrm{ml}$ calpeptin); there was no significant change in ABCA1 mRNA (not shown). These results indicate that calpain protease is a physiological regulator of ABCA1 protein turnover.

ApoA-I-mediated increase of ABCA1 is caused by inbibition of calpain degradation of $A B C A 1$. ABCA1 binds and crosslinks apoA-I $(14,18)$, raising the possibility that apoAI could modulate the turnover of ABCA1. ApoA-I increased ABCA1 protein levels in transfected 293 cells (Figure 4a), resulting in a maximal four- to fivefold induction of ABCA1 at concentrations of $10-20 \mu \mathrm{g} / \mathrm{ml}$ 


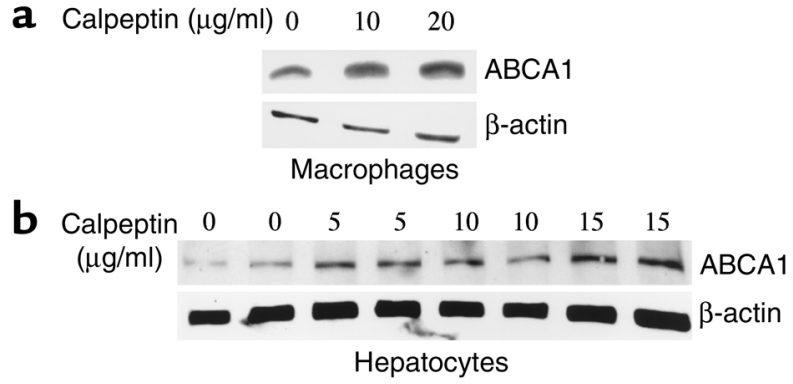

Figure 3

Calpeptin increases $A B C A 1$ in primary mouse macrophages and hepatocytes. Levels of ABCA1 in mouse peritoneal macrophages (a) or primary mouse hepatocytes (b) incubated with or without calpeptin at indicated concentration for 3 hours. The data are representative of three independent experiments with similar results.

after 2-4 hours of incubation. These concentrations of lipid-poor apoA-I are likely to be present in lymph and interstitial fluid (26). These findings are similar to those in a recent report using transformed macrophage cultures (19). As in that report, we found that stabilization of ABCA1 protein by apoA-I occurred without any change in $A B C A 1$ mRNA (not shown).

Importantly, the ability of apoA-I to increase ABCA1 protein in cultured cells was observed for wild-type ABCA1 but not for ABCA1delPEST (Figure 4b; $2.9 \pm 0.4-$ fold increase for wild-type, $n=4, P<0.01 ; 1.2 \pm 0.2$-fold increase for ABCA1delPEST, $n=4, P=0.87$ ), even though apoA-I bound and mediated lipid efflux in cells expressing ABCA1delPEST (Figure 1). This suggests that the effect of apoA-I on expression of ABCA1 is mediated via the PEST sequence. Moreover, pretreatment of cells with apoA-I abolished the ability of calpain to mediate proteolysis of $A B C A 1$ (Figure $2 \mathrm{~d}$ ), providing direct evidence to link the effect of apoA-I with calpain-mediated proteolysis. Treatment of cells with apoA-I (with or without proteasome inhibitors) did not affect ubiquitination levels of ABCA1 (not shown); this excluded decreased proteasomal degradation as a mechanism. These results suggest that the addition of apoA-I to cells activates a positive feedback loop that leads to increased levels of ABCA1 protein. This effect requires the PEST sequence in $A B C A 1$ and is mediated by decreased proteolysis of ABCA1 by calpain protease.

ApoA-I binding is required for apoA-I-mediated increase of $A B C A 1$. Next we carried out experiments to determine the mechanism by which apoA-I stabilizes ABCA1 protein. The dose responses of the apoA-I effect on ABCA1 levels (Figure $3 \mathrm{a}$ ) and on binding and cholesterol efflux (27) are similar, indicating that the effects of apoA-I could be mediated either by increased binding or by cholesterol or phospholipid efflux. To assess the role of cholesterol efflux, cells were depleted of cholesterol by incubation with cyclodextrin, to a similar level to that achieved by ABCA1 expression (Figure 5a). However, there was no increase in ABCA1 levels; this ruled out cholesterol efflux as a mechanism. Also, incubation with $\mathrm{HDL}_{2}(50 \mu \mathrm{g}$ protein $/ \mathrm{ml})$, which contains apoA-I but does not interact with ABCA1 (28), did not affect ABCA1 levels, suggesting that a direct interaction with ABCA1 is required. Mutation of the cytoplasmic ATPbinding Walker motif sequence abolishes cellular binding of apoA-I and phospholipid and cholesterol efflux $(28,29)$. In different experiments the Walker motif mutant showed variable levels of basal expression compared with wild-type, but it consistently showed no increase in ABCA1 protein levels when incubated with apoA-I (Figure $5 \mathrm{~b}$ ). Thus, the stabilization of ABCA1 is likely secondary to apolipoprotein binding and/or phospholipid efflux.

A recent study showed that a mutation of ABCA1 that causes Tangier disease (W590S) resulted in a moderate increase in apoA-I binding but defective lipid efflux (30). We tested this mutant ABCA1 for its response to apoA-I and calpeptin. As reported (30), ABCA1-W590S did not mediate cholesterol and phospholipid efflux to apoA-I and showed a slight increase in apoA-I binding when expressed in 293 cells (not shown). ApoA-I failed to increase ABCA1-W590S levels in 293 cells (Figure 5c). Similar results were obtained in three different experiments. In contrast, like wild-type ABCA1, calpeptin significantly increased ABCA1W590S levels (Figure 5c). These results suggest that apoA-I binding is required but not sufficient to induce stabilization of ABCA1. Additional signals following apoA-I binding to ABCA1 are necessary for subsequent inhibition of calpain degradation.

ApoA-I and apoE increase ABCA1 in primary macrophages and hepatocytes. ABCA1 binds other apolipoproteins in addition to apoA-I (31). To test whether the effect on ABCA1 is apoA-I- or macrophage-specific, we examined the effect of apoA-I and apoE in primary cultures of murine macrophages and hepatocytes. ApoA-I induced a marked increase in ABCA1 levels in primary macrophages (Figure 6a) and hepatocytes (Figure 6C).
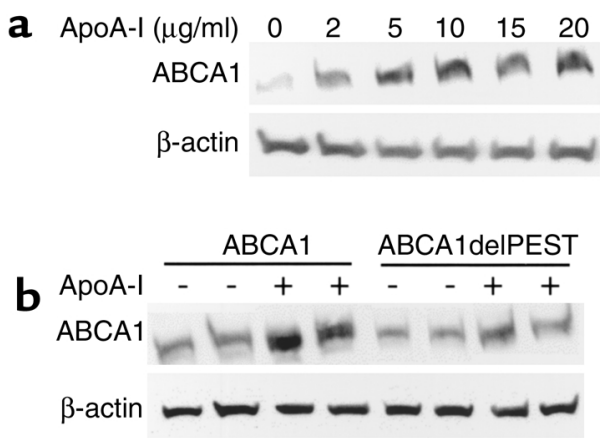

\section{Figure 4}

ApoA-I-mediated increase in ABCA1 requires PEST sequence. (a) Dose-response effect of apoA-I (3 hours' incubation) in HEK293 cells transfected with wild-type ABCA1. (b) Wild-type ABCA1 and ABCA1delPEST protein levels after 3 hours of incubation with apoA-I $(10 \mu \mathrm{g} / \mathrm{ml})$ in 293 cells transiently transfected with the FLAG-tagged constructs, as determined by Western blot with FLAG antibodies. The data are representative of three (a) or two (b) experiments with similar results. 


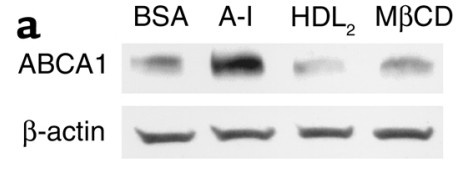

\section{b}
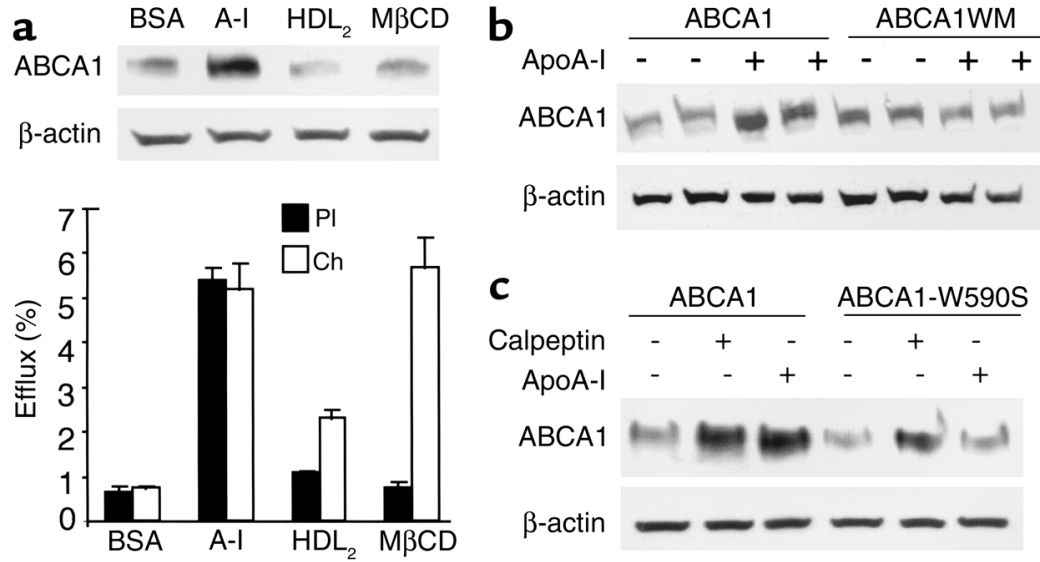
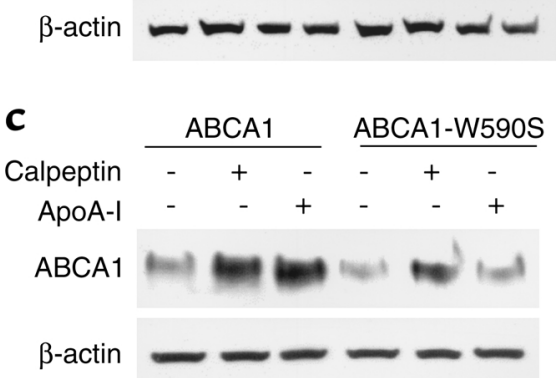

\section{Figure 5}

Effects of cholesterol and phospholipid efflux on the induction of ABCA1 by apoA-I. (a, top) $A B C A 1$ protein level in mouse peritoneal macrophages after 3 hours of incubation with $10 \mu \mathrm{g} / \mathrm{ml}$ apoA-I or $50 \mu \mathrm{g} / \mathrm{ml} \mathrm{HDL}_{2}$, or after pretreatment with $1 \mathrm{mM}$ methyl- $\beta$ cyclodextrin $(\mathrm{M} \beta \mathrm{CD})$ for 5 minutes to deplete cholesterol and then with control media for 3 hours. (a, bottom) Phospholipid (PI) and cholesterol (Ch) efflux in macrophages treatments similar to those in the top panel. (b) ABCA1-FLAG and Walker-mutant (K939M) ABCA1-FLAG (ABCA1WM) protein levels after 3 hours of incubation with apoA-I $(10 \mu \mathrm{g} / \mathrm{ml})$ in transiently transfected 293 cells. (c) Wild-type ABCA1-FLAG and ABCA1-W590S-FLAG protein levels after 3 hours of incubation with apoA-I $(10 \mu \mathrm{g} / \mathrm{ml})$ or calpeptin $(20 \mu \mathrm{g} / \mathrm{ml})$ in 293 cells. The data are representative of two (a and $\mathbf{b})$ or three (c) experiments with similar results.

The increase of ABCA1 in macrophage cell lysates was paralleled by an increase in cell surface ABCA1 (Figure $6 \mathrm{~b})$. ApoA-I caused stabilization of ABCA1 in freshly isolated hepatocytes from chow-fed mice (Figure 6c), whereas, in primary macrophages, upregulation of ABCA1 expression by pretreatment with acetyl-LDL and LXR/RXR activators was required in order to see the effect of apoA-I (Figure 6d). This likely reflects higher basal expression of ABCA1 in hepatocytes, and it is consistent with a posttranscriptional mode of regulation. ApoE is secreted by macrophages, and apoE infusion or expression in macrophages is antiatherogenic (32, 33). Incubation of macrophages with exogenous apoE increased ABCA1 protein in mouse peritoneal macrophages (Figure 6, e and f), similar to the effects of apoA-I. Moreover, in transfected 293 cells, apoE increased wild-type ABCA1 but had no effect on expression of the W590S or PEST deletion mutants of ABCA1 (Figure 6g), indicating that apoA-I and apoE regulate ABCA1 by similar mechanisms.

ApoA-I infusion increases ABCA1 in vivo. In order to determine whether apoA-I could also increase ABCA1 in vivo, we injected mice intravenously with apoA-I. This resulted in an induction of ABCA1 protein in liver and peritoneal macrophages (Figure 7, $a$ and b). The effects in liver were observed in chow-fed animals, whereas those in macrophages were seen after feeding a high-fat, high-cholesterol diet for 7 days. ApoA-I infusion did not significantly alter ABCA1 mRNA levels in liver, as determined by quantitative real-time PCR using a TaqMan probe (not shown). A time- course study of apoA-I infusion showed that the increase in ABCA1 was sustained for 8 hours, but after 24 hours ABCA1 protein levels had returned to base line, likely reflecting the rapid clearance of apoA-I from plasma. We also measured ABCA1 protein levels in liver from apoA-I transgenic ( $n=6$ mice per group) and apoA-I knockout mice ( $n=2$ mice) and found no significant difference from controls (for the wild-type mice, mean ABCA1 level normalized to actin $=0.25 \pm 0.14$; for the apoA-I transgenic, mean $=0.15 \pm 0.03$, $P=0.18$ ), suggesting that some form of chronic adaptation may occur as a result of continuous expression.

\section{Discussion}

This study reveals a novel mode of regulation of ABCA1 by calpain proteolysis, which is reversed by the extracellular ligand apoA-I acting through a PEST sequence in ABCA1. The findings appear to represent the first example of positive feedback control of a cell surface transporter in which the ligand turns off PEST sequence-regulated calpain proteolysis of the transporter. The nature of the signal that links apolipoprotein binding to proteolysis is unknown, but this process could be initiated by dissociation of phospholipid/apoA-I complexes from the transporter. The increase in ABCA1 in vivo is consistent with the hypothesis that antiatherogenic effects of apoA-I infusion are mediated by enhanced macrophage cholesterol efflux $(6,7)$ and suggests that strategies to mimic the effects of apolipoprotein binding, or to inhibit calpain proteolysis, might increase $\mathrm{ABCA} 1$ and decrease atherosclerosis.

The involvement of the PEST sequence in calpain proteolysis and apolipoprotein stabilization of ABCA1 was shown by multiple approaches. First, the major phenotypes produced by deletion of the PEST sequence or calpeptin were similar (i.e., a marked increase in cell surface ABCA1), and there was no additional increase when treatments were combined, indicating that PEST deletion and calpeptin are both acting in the same pathway. Similarly, the effect of apoA-I on ABCA1 levels was blocked by deletion of the PEST sequence. We independently linked calpain proteolysis and apolipoprotein effects to the PEST sequence by demonstrating that the degradation of ABCA1 by purified $\mu$-calpain in permeabilized cells was abolished both by the PEST deletion and by pretreatment with apoA-I. One difference between the phenotype that resulted from calpeptin or apolipoprotein treatment and the phenotype of the PEST deletion mutant was that the latter did not show an increase in total ABCA1 in cell lysates. This probably 


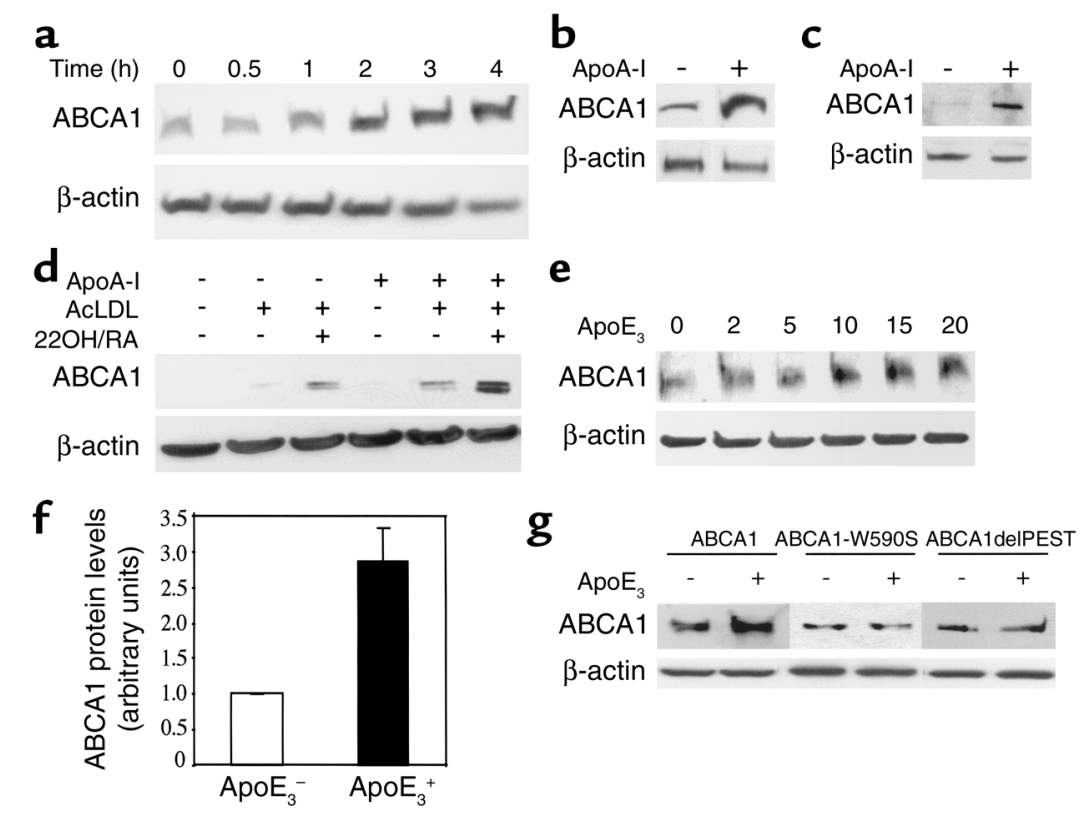

\section{Figure 6}

ApoA-I and apoE increase ABCA1 protein in mouse primary hepatocytes and macrophages. (a) Time course of the effect of apoA-I (10 $\mu \mathrm{g} / \mathrm{ml})$ on $A B C A 1$ protein levels in mouse peritoneal macrophages pretreated overnight with $50 \mu \mathrm{g} / \mathrm{ml}$ acetyl-LDL and LXR/RXR ligands $22(R)$-hydroxycholesterol and 9-cis retinoic acid (RA) (both $10 \mu \mathrm{M}$ ). (b) Cell surface biotinylated ABCA1 levels in macrophages after 3 hours of incubation with $10 \mu \mathrm{g} / \mathrm{ml}$ apoA-I. (c) ABCA1 levels in primary mouse hepatocytes incubated for 3 hours with $10 \mu \mathrm{g} / \mathrm{ml}$ apoA-I. (d) Effects of apoA-I $(10 \mu \mathrm{g} / \mathrm{ml})$ on $A B C A 1$ levels in macrophages treated with or without acetyl-LDL (AcLDL) and LXR/RXR ligands. (e) Dose response of apoE 3 effect (3 hours' incubation) on ABCA1 protein levels in mouse peritoneal macrophages pretreated with acetyl-LDL, 22(R)-hydroxycholesterol, and 9-cis retinoic acid. (f) ABCA1 levels in mouse peritoneal macrophages after 3 hours of incubation with or without apo $E_{3}(10 \mu \mathrm{g} / \mathrm{ml})$. (g) ABCA1, W590S-ABCA1, and ABCA1delPEST protein levels in HEK293 cells after 3 hours of incubation with or without apoE $E_{3}$. The data are representative of two $(\mathbf{a}, \mathbf{b}, \mathbf{e}$, and $\mathbf{g})$ or three $(\mathbf{c}, \mathbf{d}$, and $\mathbf{f})$ experiments with similar results.

reflects an additional defect in synthesis of the PEST deletion mutant, perhaps related to a nonspecific effect of the large deletion on translation or mRNA stability. Consistent with this suggestion, several point mutants in the PEST sequence have been found to increase both cell surface and total ABCA1 (not shown).

Thus, cell surface ABCA1 is regulated by an intriguing mechanism that may be more generally relevant to the regulation of cell surface transporters by calpain proteolysis. In the basal state, ABCA1 likely binds calpain via its PEST sequence, resulting in proteolysis. The interaction of $A B C A 1$ with the extracellular, lipid-binding ligand (apoA-I or apoE) leads to inhibition of calpain-mediated proteolysis and an increase in total and cell surface ABCA1. One possible mechanism is that the binding of apoA-I causes a conformational change in ABCA1 that leads to decreased binding of calpain

\section{Figure 7}

ApoA-I injection increases ABCA1 protein in hepatocytes and macrophages in mice. (a) Hepatic ABCA1 levels in mice 4 hours after intravenous injection of apoA-I ( $20 \mathrm{mg} / \mathrm{kg}$ body weight) or albumin as control (Ctr). The bar graph represents quantification of ABCA1 protein levels normalized against $\beta$-actin ( $n=5$ for each group, $P<0.01)$. (b) Macrophage ABCA1 levels in mice 4 hours after apoA-I injection $(n=5$ for apoA-I group and $n=3$ for control group, $P<0.006)$. protease to the PEST sequence. Another possibility is that these effects are brought about by phospholipid efflux mediated by apoA-I. Phospholipid efflux mediated by apoA-I might lead to a local change in membrane phospholipids that decreases the binding of a hydrophobic, glycine-rich sequence of the small calpain subunit, which stabilizes binding of calpain to membranes (34). This possibility is favored by a key mutant ABCA1 that binds apoA-I but does not mediate lipid efflux (30). ApoA-I fails to increase ABCA1-W590S (Figure 5c), implying that phospholipid efflux is required for apoA-I-mediated ABCA1 stabilization.

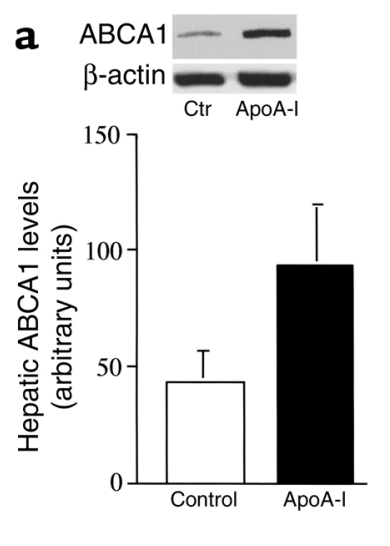


The results suggest that a signaling process may be initiated by apoA-I-mediated phospholipid efflux, leading to decreased binding of calpain to the PEST sequence. Calpain proteases comprise a small gene family with 12 identified members (35). Since the inhibition of ABCA1 degradation by calpeptin was observed in several cell types and was reproduced by adding purified $\mu$-calpain protease, it is likely to be mediated by capn 1 and capn 4 , which together form the two subunits of the widely expressed $\mu$-calpain protease (36). However, we cannot rule out the possible involvement of more specific calpains in different cell types.

While our studies were ongoing, Arakawa and Yokoyama reported the apolipoprotein stabilization of ABCA1 in cell culture (19), and our major findings are consistent with and complementary to theirs. Our study was initially focused on the role of a PEST sequence in regulating calpain proteolysis of ABCA1 and provides an important link with the earlier work (19) by showing that the apolipoprotein effects are mediated by inhibition of calpain proteolysis and require the PEST sequence. Moreover, we show the stabilization of ABCA1 by apoA-I and apoE in primary macrophage cultures, and the likely involvement of similar mechanisms for both apolipoproteins. In contrast to the earlier study (19), we showed that ABCA1 is ubiquitinated and that lactacystin increased ABCA1 in transfected 293 cells and acetyl-LDL-loaded, LXR/ RXR-activated macrophages (not shown). However, the effect of lactacystin was moderate and irrelevant to the PEST sequence-mediated calpain degradation. Moreover, expression of the $\mathrm{N}$-terminal half of ABCA1, which does not contain the PEST sequence, also resulted in cell surface expression and ubiquitination (not shown), suggesting that the ubiquitinated residues are remote from the PEST sequence. Together our studies indicate that under normal cellular conditions, ABCA1 turnover is rapid, probably primarily reflecting nonproteasomal calpain-mediated degradation. Similar to our findings, Feng and Tabas (37) found that lactacystin increased ABCA1 in macrophages loaded with acetyl-LDL and cholesterol ester (37). However, the effect of lactacystin became more pronounced in macrophages loaded with acetyl-LDL in the presence of an acyl-CoA:cholesterol acyltransferase inhibitor, leading to the suggestion that the proteasomal degradation pathway is activated in free cholesterol-loaded cells (37). Together our studies suggest that a distinct ubiquitin-proteasome degradation pathway of ABCA1 is activated in free cholesterol-loaded macrophages, a condition that eventuates in cell death (38). Thus, two different ABCA1 degradation pathways can be active under various cellular conditions: a basal calpain degradation pathway that is turned off by interaction with apolipoproteins, and a ubiquitin-proteasome pathway that is activated by marked free-cholesterol loading. There is a precedent for such dual regulation in the distinct proteasome and calpain degradation pathways of IKB (24).
Importantly, we demonstrate that ABCA1 is increased in hepatocytes and macrophages in vivo following apoA-I infusion. It could appear paradoxical that injection of apoA-I in an amount representing approximately $15 \%$ of total plasma apoA-I induces an increase in macrophage and hepatic ABCA1. However, the majority of apoA-I associated with bulk HDL is a poor substrate of ABCA1 compared with lipid-poor apoA-I (28). Injection of free apoA-I is expected to substantially increase the latter pool in plasma.

The findings of these in vivo studies may help explain the antiatherogenic effects of apoA-I and apoE infusion and help provide a rationale for apoA-I infusion trials in humans. Intravenous injection of apoA-I increased ABCA1 in liver and peritoneal macrophages. The increase in ABCA1 in macrophages is likely to lead to an increase in macrophage cholesterol efflux and to reversal of foam cell formation. While macrophage ABCA1 does not make a major contribution to plasma HDL levels (39), the stabilization of ABCA1 in liver is likely to result in an increase in HDL levels. Increased HDL levels could promote macrophage cholesterol efflux by multiple mechanisms and could also be antiatherogenic through anti-inflammatory effects of HDL (9). Unexpectedly, increases in ABCA1 protein were not found in apoA-I transgenic mice; this highlights the complexity of these models, and the likely existence of multiple antiatherogenic mechanisms operating through the HDL fraction.

ABCA1 expression is highly regulated, on both transcriptional and posttranscriptional levels. The interaction with apoA-I modulates both forms of regulation. Thus, cholesterol efflux promoted by ABCA1 leads to decreased activation of LXR/RXR by oxysterols and ultimately decreases ABCA1 transcription and protein levels, but only after cells are no longer cholesterol-loaded. In contrast, the present study indicates that apoA-I and apoE have a positive effect on ABCA1 protein expression, and this is likely to be important in hepatocytes and macrophage foam cells. Intense interest has recently centered on the possibility that increasing macrophage cholesterol efflux could represent a novel approach to treatment of atherosclerosis (9). LXR/RXR targets a battery of genes that mediate cholesterol efflux, transport, and excretion, and LXR activators are antiatherogenic (40). However, LXR/RXR also increases transcription of SREBP1c and its target genes, causing fatty liver and hypertriglyceridemia $(41,42)$. Our results suggest that calpain protease inhibitors (43), or small molecules that modulate the local interaction of ABCA1 with calpain protease at the plasma membrane, might provide an alternative way to upregulate $A B C A 1$ protein. This strategy is especially appealing since it may mimic the stabilizing effect of the natural ligands apoA-I and apoE.

\section{Acknowledgments}

This study was supported by NIH grant HL-22682. 
1. Brown, M.S., Ho, Y.K., and Goldstein, J.L. 1980. The cholesteryl ester cycle in macrophage foam cells. Continual hydrolysis and re-esterification of cytoplasmic cholesteryl esters. J. Biol. Chem. 255:9344-9352.

2. Ho, Y.K., Brown, M.S., and Goldstein, J.L. 1980. Hydrolysis and excretion of cytoplasmic cholesteryl esters by macrophages: stimulation by high density lipoprotein and other agents. J. Lipid Res. 21:391-398.

3. Oram, J.F., Albers, J.J., Cheung, M.C., and Bierman, E.L. 1981. The effects of subfractions of high density lipoprotein on cholesterol efflux from cultured fibroblasts. Regulation of low density lipoprotein receptor activity. J. Biol. Chem. 256:8348-8356.

4. Rubin, E.M., Krauss, R.M., Spangler, E.A., Verstuyft, J.G., and Clift, S.M 1991. Inhibition of early atherogenesis in transgenic mice by human apolipoprotein AI. Nature. 353:265-267.

5. Plump, A.S., Scott, C.J., and Breslow, J.L. 1994. Human apolipoprotein A-I gene expression increases high density lipoprotein and suppresses atherosclerosis in the apolipoprotein E-deficient mouse. Proc. Natl. Acad. Sci. USA. 91:9607-9611.

6. Chiesa, G., et al. 2002. Recombinant apolipoprotein A-I(Milano) infusion into rabbit carotid artery rapidly removes lipid from fatty streaks. Circ. Res. 90:974-980.

7. Eriksson, M., Carlson, L.A., Miettinen, T.A., and Angelin, B. 1999. Stimulation of fecal steroid excretion after infusion of recombinant proapolipoprotein A-I. Potential reverse cholesterol transport in humans. Circulation. 100:594-598.

8. Cole, T.G., Nowatzke, W.L., Bisgaier, C.L., and Krause, B.R. 2002 Method-dependent changes in "HDL-cholesterol" with recombinant apolipoprotein A-I(Milano) infusion in healthy volunteers. Clin. Chem. 48:680-681.

9. Lusis, A.J. 2000. Atherosclerosis. Nature. 407:233-241.

10. Tall, A.R., and Wang, N. 2000. Tangier disease as a test of the reverse cholesterol transport hypothesis. J. Clin. Invest. 106:1205-1207.

11. Schaefer, E.J., Zech, L.A., Schwartz, D.E., and Brewer, H.B., Jr. 1980. Coronary heart disease prevalence and other clinical features in familial highdensity lipoprotein deficiency (Tangier disease). Ann. Intern. Med. 93:261-266.

12. Brooks-Wilson, A., et al. 1999. Mutations in ABC1 in Tangier disease and familial high-density lipoprotein deficiency. Nat. Genet. 22:336-345.

13. Attie, A.D., Kastelein, J.P., and Hayden, M.R. 2001. Pivotal role of ABCA1 in reverse cholesterol transport influencing HDL levels and susceptibility to atherosclerosis. J. Lipid Res. 42:1717-1726.

14. Wang, N., Silver, D.L., Costet, P., and Tall, A.R. 2000. Specific binding of ApoA-I, enhanced cholesterol efflux, and altered plasma membrane morphology in cells expressing ABC1. J. Biol. Chem. 275:33053-33058.

15. Repa, J.J., et al. 2000. Regulation of absorption and ABC1-mediated efflux of cholesterol by RXR heterodimers. Science. 289:1524-1529.

16. Costet, P., Luo, Y., Wang, N., and Tall, A.R. 2000. Sterol-dependent transactivation of the $\mathrm{ABC} 1$ promoter by the liver $\mathrm{X}$ receptor/retinoid $\mathrm{X}$ receptor. J. Biol. Chem. 275:28240-28245.

17. Langmann, T., et al. 1999. Molecular cloning of the human ATP-binding cassette transporter 1 (hABC1): evidence for sterol-dependent regulation in macrophages. Biochem. Biophys. Res. Commun. 257:29-33.

18. Oram, J.F., Lawn, R.M., Garvin, M.R., and Wade, D.P. 2000. ABCA1 is the cAMP-inducible apolipoprotein receptor that mediates cholesterol secretion from macrophages. J. Biol. Chem. 275:34508-34511.

19. Arakawa, R., and Yokoyama, S. 2002. Helical apolipoproteins stabilize ATP-binding cassette transporter A1 by protecting it from thiol protease-mediated degradation. J. Biol. Chem. 277:22426-22429.

20. Honkakoski, P., and Negishi, M. 1998. Protein serine/threonine phosphatase inhibitors suppress phenobarbital-induced Cyp2b10 gene transcription in mouse primary hepatocytes. Biochem. J. 330:889-895.

21. Rechsteiner, M., and Rogers, S.W. 1996. PEST sequences and regulation by proteolysis. Trends Biochem. Sci. 21:267-271.
22. WWW PESTfind Analysis. European Molecular Biology Network. http://at.embnet.org/embnet/tools/bio/PESTfind/.

23. Roth, A.F., Sullivan, D.M., and Davis, N.G. 1998. A large PEST-like sequence directs the ubiquitination, endocytosis, and vacuolar degradation of the yeast a-factor receptor. J. Cell Biol. 142:949-961.

24. Shumway, S.D., Maki, M., and Miyamoto, S. 1999. The PEST domain of IkappaBalpha is necessary and sufficient for in vitro degradation by mucalpain. J. Biol. Chem. 274:30874-30881.

25. Tsujinaka, T., et al. 1988. Synthesis of a new cell penetrating calpain inhibitor (calpeptin). Biochem. Biophys. Res. Commun. 153:1201-1208.

26. Nanjee, M.N., Cooke, C.J., Olszewski, W.L., and Miller, N.E. 2000. Concentrations of electrophoretic and size subclasses of apolipoprotein AI-containing particles in human peripheral lymph. Arterioscler. Thromb. Vasc. Biol. 20:2148-2155.

27. Chen, W., et al. 2001. Preferential ATP-binding cassette transporter A1mediated cholesterol efflux from late endosomes/lysosomes. J. Biol. Chem. 276:43564-43569.

28. Wang, N., Silver, D.L., Thiele, C., and Tall, A.R. 2001. ATP-binding cassette transporter $\mathrm{A} 1$ (ABCA1) functions as a cholesterol efflux regulatory protein. J. Biol. Chem. 276:23742-23747.

29. Marguet, D., Luciani, M.F., Moynault, A., Williamson, P., and Chimini, G. 1999. Engulfment of apoptotic cells involves the redistribution of membrane phosphatidylserine on phagocyte and prey. Nat. Cell Biol. 1:454-456.

30. Fitzgerald, M.L., et al. 2002. Naturally occurring mutations in ABCA1's largest extracellular loops can disrupt its direct interaction with apolipoprotein A-I. J. Biol. Chem. 277:33178-33187.

31. Remaley, A.T., et al. 2001. Apolipoprotein specificity for lipid efflux by the human ABCAI transporter. Biochem. Biophys. Res. Commun. 280:818-823.

32. Mahley, R.W., et al. 1989. Intravenous infusion of apolipoprotein E accelerates clearance of plasma lipoproteins in rabbits. J. Clin. Invest. 83:2125-2130.

33. Bellosta, S., et al. 1995. Macrophage-specific expression of human apolipoprotein E reduces atherosclerosis in hypercholesterolemic apolipoprotein E-null mice. J. Clin. Invest. 96:2170-2179.

34. Imajoh, S., Kawasaki, H., and Suzuki, K. 1986. The amino-terminal hydrophobic region of the small subunit of calcium-activated neutral protease (CANP) is essential for its activation by phosphatidylinositol. J. Biochem. (Tokyo). 99:1281-1284.

35. Sorimachi, H., and Suzuki, K. 2001. The structure of calpain. J. Biochem. (Tokyo). 129:653-664.

36. Suzuki, K. 1991. Nomenclature of calcium dependent proteinase. Biomed. Biochim. Acta. 50:483-484.

37. Feng, B., and Tabas, I. 2002. ABCA1-mediated cholesterol efflux is defective in free cholesterol-loaded macrophages. Mechanism involves enhanced ABCA1 degradation in a process requiring full NPC1 activity. J. Biol. Chem. 277:43271-43280.

38. Yao, P.M., and Tabas, I. 2000. Free cholesterol loading of macrophages induces apoptosis involving the fas pathway.J. Biol. Chem. 275:23807-23813.

39. Haghpassand, M., Bourassa, P.A., Francone, O.L., and Aiello, R.J. 2001. Monocyte/macrophage expression of ABCA1 has minimal contribution to plasma HDL levels. J. Clin. Invest. 108:1315-1320. doi:10.1172/ JCI200112810

40. Joseph, S.B., et al. 2002. Synthetic LXR ligand inhibits the development of atherosclerosis in mice. Proc. Natl. Acad. Sci. USA. 99:7604-7609.

41. Repa, J.J., et al. 2000. Regulation of mouse sterol regulatory elementbinding protein-1c gene (SREBP-1c) by oxysterol receptors, LXRalpha and LXRbeta. Genes Dev. 14:2819-2830.

42. Schultz, J.R., et al. 2000. Role of LXRs in control of lipogenesis. Genes Dev. 14:2831-2838.

43. Stracher, A. 1999. Calpain inhibitors as therapeutic agents in nerve and muscle degeneration. Ann. NY Acad. Sci. 884:52-59. 